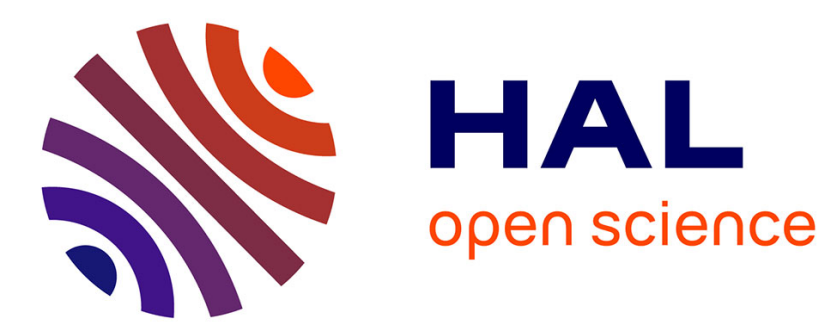

\title{
Pinning of charge density waves in irradiated blue bronzes K 0.30MoO3 and Rb0.30MoO3
}

\author{
H. Mutka, S. Bouffard, J. Dumas, C. Schlenker
}

\section{To cite this version:}

H. Mutka, S. Bouffard, J. Dumas, C. Schlenker. Pinning of charge density waves in irradiated blue bronzes K 0.30MoO3 and Rb0.30MoO3. Journal de Physique Lettres, 1984, 45 (14), pp.729-736. 10.1051/jphyslet:019840045014072900 . jpa-00232404

\section{HAL Id: jpa-00232404 https://hal.science/jpa-00232404}

Submitted on 1 Jan 1984

HAL is a multi-disciplinary open access archive for the deposit and dissemination of scientific research documents, whether they are published or not. The documents may come from teaching and research institutions in France or abroad, or from public or private research centers.
L'archive ouverte pluridisciplinaire HAL, est destinée au dépôt et à la diffusion de documents scientifiques de niveau recherche, publiés ou non, émanant des établissements d'enseignement et de recherche français ou étrangers, des laboratoires publics ou privés. 
Classification

Physics Abstracts

$72.20 \mathrm{H}-72.80 \mathrm{G}$

\title{
Pinning of charge density waves in irradiated blue bronzes $\mathrm{K}_{0.30} \mathrm{MoO}_{3}$ and $\mathrm{Rb}_{0.30} \mathrm{MoO}_{3}$
}

\author{
H. Mutka (*), S. Bouffard \\ Section d'Etude des Solides Irradiés, Centre d'Etudes Nucléaires, B.P. 6. \\ 92260 Fontenay aux Roses, France
}

\author{
J. Dumas and C. Schlenker \\ Laboratoire d'Etudes des Propriétés Electroniques des Solides, CNRS, B.P. 166, \\ 38042 Grenoble Cedex, France
}

(Reçu le 9 mars 1984, révisé le 10 mai, accepté le 30 mai 1984)

\begin{abstract}
Résumé. - On a étudié, en fonction de la concentration des défauts créés par irradiation aux électrons, la conductivité non linéaire due au dépiégeage des ondes de densité de charge dans la phase semiconductrice des bronzes bleus $\mathrm{K}_{0,30} \mathrm{MoO}_{3}$ et $\mathrm{Rb}_{0,30} \mathrm{MoO}_{3}$. Le champ électrique seuil de nonlinéarité augmente linéairement avec la concentration ce qui indique que ces défauts sont des centres de piégeage fort. Les propriétés d'hystérésis en champ électrique sont aussi modifiées par ces défauts.

Abstract. - Non linear conductivity due to the depinning of charge density waves has been studied in the Peierls distorted state of the blue bronzes $\mathrm{K}_{0.30} \mathrm{MoO}_{3}$ and $\mathrm{Rb}_{0.30} \mathrm{MoO}_{3}$ as a function of concentration of defects created by electron irradiation. The threshold electric field for the onset of non linearity is found to increase linearly with the defect concentration, as expected for strong pinning centres. The hysteretic properties are also modified by the irradiation centres.
\end{abstract}

\section{Introduction.}

Non linear transport in quasi one dimensional inorganic transition metal compounds is presently extensively studied. These properties are now well established for the trichalcogenides $\mathrm{NbSe}_{3}$, $\mathrm{TaS}_{3}[1,2]$, the molybdenum blue bronzes $\mathrm{K}_{0.30} \mathrm{MoO}_{3}$ and $\mathrm{Rb}_{0.30} \mathrm{MoO}_{3}[3,4]$ and the tetrachalcogenides such as $\left(\mathrm{TaSe}_{4}\right)_{2} \mathrm{I}$ [5]. These compounds are quasi one dimensional conductors which undergo below a critical temperature a transition towards a (usually) incommensurate charge density wave (CDW) state. The onset of non linear conductivity is attributed to the depinning of the CDW by electric fields larger than a threshold value $E_{\mathrm{t}}$. In the non linear regime, an anomalously large noise voltage including well-defined frequencies and their harmonics are also found. The existence of hysteresis and long relaxation times related to metastability has also

(*) Present address : Reactor Laboratory, Technical Research Centre of Finland, SF-02150 Espoo 15, Finland. 
now been established $[6,7]$. Some of these properties have been previously described either by considering the CDW as a classical particle moving in a periodic pinning potential $[1,8]$ or with a model of macroscopic quantum tunnelling of the CDW through potential barriers [9]. It has also been proposed that the voltage oscillations (frequency in the noise) are induced locally at the contacts and may be due to a sheet of phase vortices in the CDW lattice [10]. Recent theories take now into account the possibility of some deformation of the CDW and therefore of metastable states [11, 12]. These models could therefore describe the hysteresis, the switching found in some cases between the ohmic and the non-ohmic regime and other time dependent or memory properties $[4,6]$. In this context, the existence of CDW domains and domain boundaries has been proposed $[1,7]$. Some authors have pointed out the analogies with other physical systems such as Josephson coupled granular superconductor [13], magnetic materials [7] either in relation with the motion of a Bloch wall in a ferromagnet [4] or with spin glasses [14].

The blue bronzes have been shown to be at room temperature quasi one dimensional metals as a consequence of the presence in the crystal structure of infinite chains of $\mathrm{MoO}_{6}$ octahedra parallel to the direction of highest conductivity (monoclinic $b$-axis) $[15,16]$. The metal-semiconductor transition which takes place at $180 \mathrm{~K}$ [17] has been shown by X-ray studies to be a Peierls transition. At room temperature, precursor diffuse scattering is consistent with quasi one dimensional fluctuations. At $T<180 \mathrm{~K}$, this diffuse scattering condense into weak satellites, with an incommensurate wave vector component along $b\left(q_{b}=0.74 b^{*}\right.$ at $\left.110 \mathrm{~K}\right)$ [18]. In the incommensurate phase, sharp threshold fields are found to be in the range of $100 \mathrm{mV} / \mathrm{cm}$ and correspond in some cases to sharp switching from the ohmic to the non-ohmic regime. For $E>E_{\mathrm{t}}$, the noise voltage includes oscillations with frequencies in the range of $10 \mathrm{kHz}$. In addition to these properties, similar to those of the trichalcogenides, the blue bronzes show slow voltage pulses with pseudofrequencies in the range of $1 \mathrm{~Hz}$, either as precursors to the switching or as part of the noise for $E>E_{\mathrm{t}}[3,7]$. Up to now, these slow and partly coherent pulses seem to be typical of CDW transport in the blue bronzes. Also either pure or doped samples show in some cases slow oscillations of voltage, more or less coherent depending on the cooling process $[4,19]$.

The values of the threshold field and very likely the CDW domain configuration must be related to the crystallographic quality and the chemical purity of the samples. Previous studies on doped or on irradiated trichalcogenides clearly show the importance of crystal defects for the threshold values [20,21]. In this paper, we report the effect of electron irradiation induced defects on the pinning of CDW in the blue bronzes $\mathrm{K}_{0.30} \mathrm{MoO}_{3}$ and $\mathrm{Rb}_{0.30} \mathrm{MoO}_{3}$. These results establish that these defects are strong pinning centres. They also corroborate the importance of purity in the metastability.

\section{Experiment.}

The single crystals used in this study are synthetized as described elsewhere [16]. They can easely be cleaved along the $(\overline{2} 01)$ plane. Standard four-probe resistivity measurements were performed along the highly conducting $b$-axis. Contacts were made by indium soldering of gold leads on evaporated indium stripes. As previously reported [22], the metal-semiconductor transition was found for both compounds, on the basis of low field dc resistivity measurements, in the vicinity of $180 \mathrm{~K}$. The differential resistance $\mathrm{d} V / \mathrm{d} I$ was obtained as a function of the dc current by phase locked detection of the response to a dc and a superimposed ac excitation (at a frequency of $770 \mathrm{~Hz}$, with an ac current smaller than $10^{-2}$ times the threshold value of the dc current). The measurements were carried out either in liquid nitrogen or in gaseous hydrogen at temperatures ranging from 60 to $120 \mathrm{~K}$. Both methods gave identical results at $78 \mathrm{~K}$, indicating that self-heating of samples is negligible in gaseous hydrogen.

The crystals were irradiated with $2.5 \mathrm{MeV}$ electrons in gaseous hydrogen at temperatures between $65 \mathrm{~K}$ and $75 \mathrm{~K}$. A preliminary study of the energy dependence of the damage production rate suggests that displacement of atoms through elastic collisions is the main process for the 
defect production. It is likely that with $2.5 \mathrm{MeV}$ electrons, all three atoms species are involved in the displacement cascades. It is difficult to evaluate the displacement threshold energies and the defect concentration. However, an indication of this concentration is obtained by measuring the d-electron paramagnetic resonance signal. Non irradiated samples show several EPR centres related to deviation from stoichiometry and attributed to $\mathrm{Mo}^{5+}\left(4 \mathrm{~d}^{1}\right)$ ions, with concentrations in the range of $10^{-5}$ the total number of Mo sites [23]. The intensity of an EPR line increases after irradiation. The Curie law character of this line allows us a direct determination of a number of non-interacting spins. For an irradiation dose of $1 \mathrm{mC} / \mathrm{cm}^{2}$ at $2.5 \mathrm{MeV}$, the excess defect concentration is found to be also of the order of $10^{-5}$, after a room temperature annealing. The defects created at low temperature $(\sim 70 \mathrm{~K})$ in blue bronzes relax to a more stable configuration when the temperature exceeds $150 \mathrm{~K}$, so the defect concentration active for the conductivity is expected to be 4 or 5 times larger before this annealing.

Low field resistivity measurements have indicated that the Peierls transition temperature is insensitive to the defect concentration induced by irradiation doses used in this study and smaller that $6 \mathrm{mC} / \mathrm{cm}^{2}$. It is therefore reasonable to assume that the CDW distortion amplitude at a given temperature is approximately independent of this concentration, for the experiments reported here. At higher doses, a decrease of the Peierls transition temperature is observed when the degree of disorder increases.

Figure 1 shows typical results for the differential resistance $\mathrm{d} V / \mathrm{d} I$ as a function of the dc current $I$, obtained on a $\mathrm{Rb}_{0.30} \mathrm{MoO}_{3}$ single crystal, at several temperatures before irradiation and at $60 \mathrm{~K}$ after a small irradiation dose of $18 \mu \mathrm{C} / \mathrm{cm}^{2}$. These data were obtained with a sweep time for a $V-I$ cycle of approximatively $2 \mathrm{~min}$. However the shape of the curve and the threshold values were found to be the same for faster sweep rates (down to $20 \mathrm{~s}$ for a cycle). These data show that in the explored temperature range, the threshold field increases with temperature. Also the qualitative features of these curves depend on temperature : hysteresis appears below $110 \mathrm{~K}$ and it should be noted that it involves also the ohmic resistivity and not only the non-ohmic one. The data obtained at $77 \mathrm{~K}$ indicate that for this sample, there is no hysteresis when the current is swept without being reversed. At lower temperatures, $(T \lesssim 70 \mathrm{~K})$, slow frequency pulses are detected. The bottom curve shows that, in the case of this sample, at $60 \mathrm{~K}$ an irradiation dose corresponding to a defect concentration smaller than $10^{-5}$ induces approximately a doubling of $E_{\mathrm{t}}$ and at the same time changes the onset of non linearity from a smooth to a switching process. It should also be noted that smaller switchings are found in the so-called ohmic regime.

Figure 2 shows the threshold field as a function of temperature (logarithmic scales) before and after several irradiation doses for the same $\mathrm{Rb}_{0.30} \mathrm{MoO}_{3}$ sample. In all cases, $E_{\mathrm{t}}$ has been defined as the value obtained with decreasing dc current, corresponding to a steep change in $\mathrm{d} V / \mathrm{d} I$ when such a change was found. $E_{\mathrm{t}}$ is always increasing with temperature between $60 \mathrm{~K}$ and $120 \mathrm{~K}$, more and more steeply when the defect concentration becomes larger. The effect of an annealing at room temperature is also reported. The defect rearrangement decreases the threshold field by a factor of about 1.5. $E_{\mathrm{t}}$ is found to increase by several orders of magnitude as a function of the irradiation dose, as better shown on figure 3. The increase of $E_{\mathrm{t}}, E_{\mathrm{t}}(c)-E_{\mathrm{t}}(0)$, is plotted as a function of the dose for two samples, one of $\mathrm{K}_{0.30} \mathrm{MoO}_{3}$ and the other of $\mathrm{Rb}_{0.30} \mathrm{MoO}_{3}$. The value of the threshold field is sometimes not well defined, especially when the $V-I$ curves show several breaks. In these cases, we have reported the different possible values of $E_{\mathrm{t}}$. All data are consistent with a unique straight line, corresponding to a linear increase of $E_{\mathrm{t}}$ with the defect concentration. This result therefore does not seem to depend on the nature of the alcaline metal.

Figure 4, showing the differential resistance as a function of the dc current $I$ after a total irradiation dose of $480 \mu \mathrm{C} / \mathrm{cm}^{2}$, emphasizes the importance of metastability, especially for the ohmic resistance. The irradiation increases the ohmic resistance by a factor of 2 , however the first current cycle restores the initial value of the resistance. A current cycle which does not exceed the threshold field does not change the ohmic resistance : the decrease of the ohmic resistance is related to the 


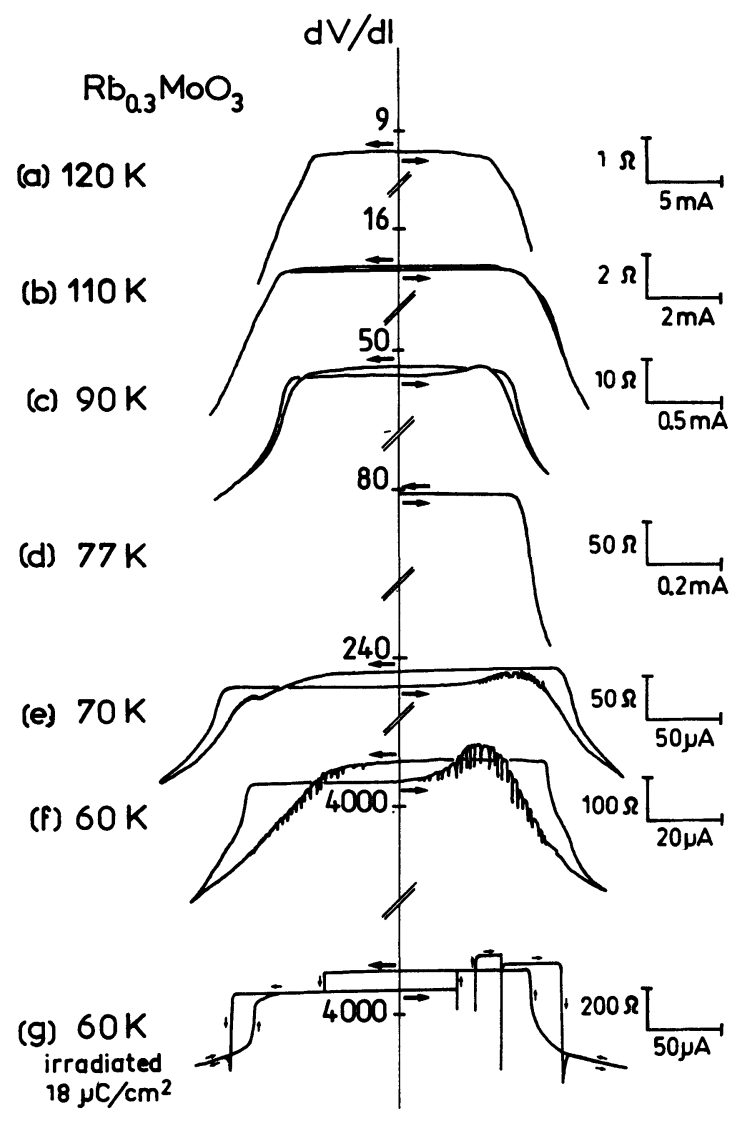

Fig. 1. - Differential resistance $d V / d I$ as a function of the dc current $I$ for a crystal of $R_{0.30} \mathbf{M o O}_{3}$. The scales, shown on the right, are different for all the curves.

crossing of the threshold field. One should note that during this first cycle (curve 1), a non-linearity appears at small $I$, followed by a large switching at higher currents. The curves (2) and (3) are then reproducible upon current cycling.

\section{Discussions.}

Let us first note that the crystals of both $\mathrm{K}_{0.30} \mathrm{MoO}_{3}$ and $\mathrm{Rb}_{0.30} \mathrm{MoO}_{3}$ may be separated in two classes according to the non linear transport, probably in relation with the crystal quality and purity. Some of them (about $10 \%$ ) show a switching from the ohmic to the non ohmic regime, while the others show a smooth threshold, without break in the $V(I)$ (or $\mathrm{d} V / \mathrm{d} I$ vs. $I$ ) characteristic. The $\mathrm{Rb}_{0.30} \mathrm{MoO}_{3}$ sample used in this study did not show, in the virgin state, a switching.

Figure 1 shows clearly that for this sample, even in the virgin state, there is at low dc current and for $T \lesssim 110 \mathrm{~K}$ a noticeable hysteresis associated to the ohmic resistivity. Similar results have also been found by Higgs and Gill in orthorhombic $\mathrm{TaS}_{3}$ [6]. This must be due to single 


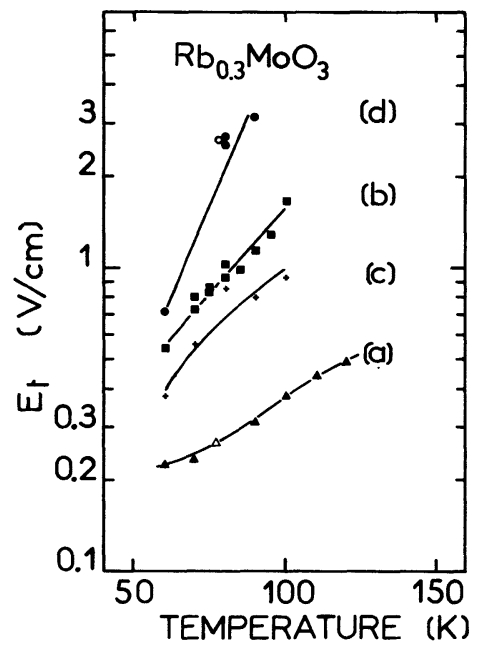

Fig. 2. - Threshold field $E_{\mathrm{t}}$ as a function of temperature for the same crystal of $\mathrm{Rb}_{0.30} \mathrm{MoO}_{3}$. (a) Virgin state. (b) After an irradiation with electrons of $2.5 \mathrm{MeV}$ and for a dose of $91 \mu \mathrm{C} / \mathrm{cm}^{2}$. (c) After the same irradiation followed by an annealing at room temperature. (d) After an irradiation dose of $480 \mu \mathrm{C} / \mathrm{cm}^{2}$ followed by an annealing at room temperature. Data obtained in gaseous hydrogen except for $\Delta$ and 0 , obtained in liquid nitrogen.

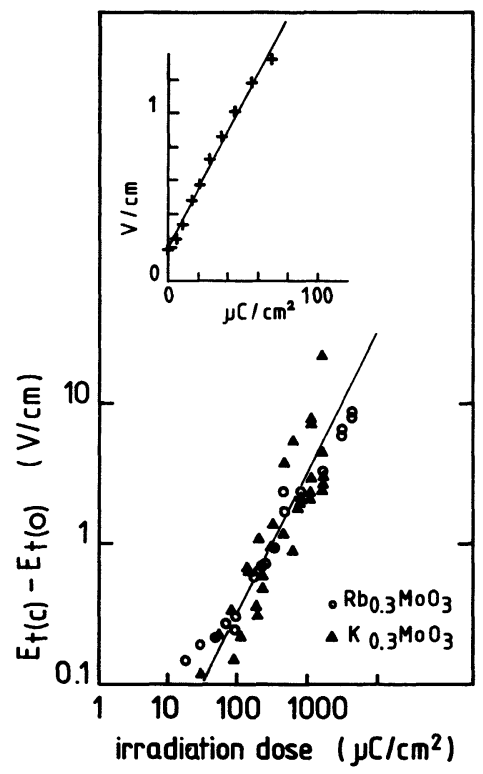

Fig. 3. - Increase in the threshold field $E_{1}(c)-E_{1}(0)$ as a function of the irradiation dose for the same crystal of $\mathrm{Rb}_{0.30} \mathrm{MoO}_{3}$ and a crystal of $\mathrm{K}_{0.30} \mathrm{MoO}_{3} . T \simeq 77 \mathrm{~K}$. Logarithmic scales. The straight line corresponds to a linear law. $E_{\mathrm{t}}(0) \simeq 200 \mathrm{mV} / \mathrm{cm}$ for both crystals. The insert shows the linear increase of $E_{\mathrm{t}}$ as a function of the dose for an other crystal of $\mathrm{K}_{0.30} \mathrm{MoO}_{3}$ (linear scales). 


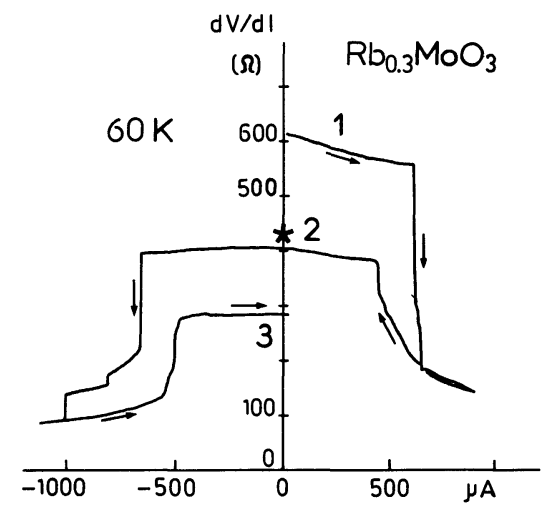

Fig. 4. - Differential resistance as a function of $I$ after a dose of $480 \mu \mathrm{C} / \mathrm{cm}^{2}$ for the same sample of $\mathrm{Rb}_{0.30} \mathrm{MoO}_{3} .(*):$ Ohmic resistivity before irradiation, the initial state after irradiation is modified by the tirst current cycle (1). Curves (2) and (3) are reproducible.

electron processes. In the case of the blue bronzes, the ohmic resistivity in the semiconducting phase is very likely extrinsic and due to non stoichiometry or impurities giving rise to acceptor or donor levels in the Peierls gap [16]. Therefore, any change in the ohmic resistivity should be related to some modifications of these levels and not to intrinsic variations of the Peierls gap. One has to conclude that in the course of dc current cycling, vacancies or impurities may be in two (or more than two) different configurations, which leads to several energy levels in the gap. This is possible if these defects may jump between neighbouring nearly equivalent sites. Such a process may be related to some kind of domain structure for the CDW lattice. It is tempting to propose that the coupling of crystal defects with domain boundaries is involved, as suggested by the analogy with the motion of a ferromagnetic domain wall in an FeSi crystal containing carbon impurities $[4,24]$. In such a picture the changes of the ohmic resistivity may also reflect a redistribution of CDW domains sizes and boundaries.

The increase found for the threshold field with temperature $(T<120 \mathrm{~K})$ (Fig. 2a) for this sample of $\mathrm{Rb}_{0.30} \mathrm{MoO}_{3}$ should be compared with similar results obtained for $\mathrm{K}_{0.30} \mathrm{MoO}_{3}$ at $T<100 \mathrm{~K}$ [3]. It is difficult to discuss these data at present as the temperature dependence of the $q$-vector is not known for our samples.

The creation of irradiation induced defects in this sample now has two main consequences, first to increase drastically the threshold field and secondly to change the qualitative features of the non linear characteristic. For the first point, $E_{1}$ is found to increase approximately linearly with the irradiation dose, and therefore with the defect concentration. This indicates that irradiation defects created in the blue bronzes, are strong pinning centres [25]. In the corresponding theoretical model, the pinning energy, or the energy gained by fixing the CDW phase at the defect sites, overcomes the elastic deformation energy associated to the CDW state. Such a linear law has already been found in $\mathrm{NbSe}_{3}$ and $\mathrm{TaS}_{3}[20,21]$. One should also note that no difference can be detected for the irradiation effects between $\mathrm{K}_{0.30} \mathrm{MoO}_{3}$ and $\mathrm{Rb}_{0.30} \mathrm{MoO}_{3}$. This indicates that the alcaline metal plays a secondary role in this process and that the active defects may be Mo vacancies on the Mo sites involved in the infinite chains of $\mathrm{MoO}_{6}$ octahedra parallel to $b$ (sites 2 and 3, see Ref. [15]). One should finally compare these data with the values of $E_{\mathrm{t}}$ obtained on Fedoped $\mathrm{K}_{0.30} \mathrm{MoO}_{3}[4]$ indicating that chemical impurities are comparatively weak pinning centres, in spite of the non isoelectronic charge of the dopant ions.

The experimental behaviours on doped or irradiated $\mathrm{NbSe}_{3}, \mathrm{TaS}_{3}$ and $\mathrm{K}_{0.30} \mathrm{MoO}_{3}$ seems to be a general result : the irradiation induced defects act as strong pinning centres while the impurities should be considered as weak pinning centres. 
The second effect of the irradiation is to change the smooth onset of non linearity into a switching process. To understand this point, one should first clarify the possible origins of different threshold behaviours. They must be related to the nature and distribution of the pinning centres. We suggest that a smooth threshold is due to the existence of several types of pinning centres with different pinning energies, possibly inhomogeneously spatially distributed. This will probably lead to CDW domains of various sizes, more or less rigid under the electric field. In the case of an abrupt threshold, one may expect on the contrary a smaller number of species of pinning centres, homogeneously spatially distributed. The CDW domains should then be more regular and possibly more strongly coupled, thus giving rise to a more collective behaviour and to a switching process. In this context, our data indicate that, the sample used in this study contained in the virgin state a small number of pinning centres with some broad spatial and energy distribution. These centres are somewhat hidden out by the centres created by irradiation and inducing a strong pinning. The irradiation must then lead to a few types of active centres, homogeneously distributed in the crystal.

The effect of a current cycle on the ohmic resistance are more surprising. The first dc current cycle anneals the increase of resistivity due to radiation damage. Moreover the depinning of CDW changes the ohmic resistance. This may indicate the pinning centres and/or the domain boundaries tend to relax towards more stable configurations when the CDW glide. The same centres seem to account for the value of the threshold field and of the ohmic resistivity. This corroborates the extrinsic nature of the ohmic resistivity in the low temperature phase of the blue bronze.

\section{Conclusion.}

These results show that irradiation induced defects act as strong pinning centres in the blue bronzes, just as in the transition metal trichalcogenides. They also corroborate the importance of metastability in these materials and the relation between the hysteretic properties and the nature of the pinning centres.

Note.

During the course of this work, C. H. Chen et al. (Phys. Rev. B 29 (1984) 3763) in a study of electron beam irradiation effects on the superlattice spots, have also shown that $\mathrm{K}_{0.30} \mathrm{MoO}_{3}$ was highly sensitive to electron irradiation. These results are complementary of our data.

\section{Acknowledgments.}

The authors wish to thank J. Marcus for the crystal growth, M. Sanquer for the E.S.R. measurements and B. Barbara for helpful discussions.

\section{References}

[1] See for example : Monceau, P., Richard, J. and Renard, M., Phys. Rev. B 25 (1982) 931 and references therein.

[2] See also : Proceedings of the International Symposium on Non linear transport and related phenomena in inorganic quasi one dimensional conductors Sapporo, Japan (Oct. 1983).

[3] Dumas, J., Schlenker, C., Marcus, J. and Buder, R., Phys. Rev. Lett. 10 (1983) 757.

[4] Dumas, J. and Schlenker, C., in Ref. [2], p. 198.

[5] Wang, Z. Z., Saint-Lager, M. C., Monceau, P., Renard, M., Gressier, P., Meerschaut, A., GueMAS, L. and RouXel, J., Solid State Commun. 45 (1983) 885. 
[6] Higgs, A. W. and Gill, J. C., Solid State Commun. 47 (1983) 737 and in Ref. [2].

See also Hutiray, Gy., Mihály, G., Mihály, L., Solid State Commun. 47 (1983) 121.

[7] Dumas, J. and Schlenker, C., Solid State Commun. 45 (1983) 885.

[8] Gruner, G., Zawadowski, A. and Chaikin, P. M., Phys. Rev. Lett. 46 (1981) 511.

[9] Bardeen, J., Phys. Rev. Lett. 24 (1980) 1978 and in Ref. [2].

[10] ONG, N. P. and Verma, G., Phys. Rev. B 27 (1983) 4495 and in Ref. [2].

[11] FISHER, D. S., Phys. Rev. Lett. 50 (1983) 1486.

[12] Le Daeron, P. Y. and Aubry, S., J. Phys. C. 16 (1983) 4827.

Matsukawa, $H$. and Takayama, $H$. in Ref. [2] and submitted to Solid State Commun.

[13] Zettl, A. and Gruner, G., Phys. Rev. B 26 (1982) 2298.

[14] Fleming, R. M. and Schneemeyer, L. F., Phys. Rev. B 28 (1983) 6996.

[15] Graham, J. and WadSley, A. D., Acta Crystallogr. 20 (1966) 93.

Ghedira, M., Thèse de Doctorat d'Etat, Université Scientifique et Médicale de Grenoble (1983).

[16] Brusetti, R., Chakraverty, B. K., Devenyi, J., Dumas, J. and Schlenker, C., in Recent Developments in Cond. Matter Phys., Eds. Devreese, J. T., Lemmens, L. F., Van Doren, V. E. and Van Royen, J. (Plenum) 1981.

[17] Fogle, W. and Perlstein, J. H., Phys. Rev. B 6 (1972) 1402.

[18] Pouget, J. P., Kagoshima, S., Marcus, J. and Schlenker, C., J. Physique Lett. 44 (1983) L-113.

Sato, M., Fujishita, H. and Hoshino, S., J. Phys. C 16 (1983) L 877.

[19] Dumas, J., Arbaoui, A., Guyot, H. and Schlenker, C., submitted to Phys. Rev. B.

[20] Brill, J. W., Ong, N. P., Eckert, J. C., Savage, J. W., Khanna, S. K. and Somoano, R. B., Phys. Rev. $B 23$ (1981) 1517.

[21] Mutka, H., Bouffard, S., Mihály, G. and Mihály, L., J. Physique Lett. 45 (1984) L-113.

Monceau, P., Richard, J. and Lagnier, R., J. Phys. C. 14 (1981) 2995.

Fuller, W. W., Gruner, G., Chaikin, P. M. and Ong, N. P., Phys. Rev. B 23 (1981) 6259.

[22] Schlenker, C., Escribe-Filippini, C., Marcus, J., Dumas, J., Pouget, J. P. and Kagoshima, S., J. Physique Colloq. 44 (1983) C3-1757.

[23] Bang, G. and SPERLich, G., Z. Phys. B 22 (1975) 1.

[24] Cotillard, J. C., Grillet, D. and Porteseil, J. L., Phys. Lett. A 88 (1982) 219.

[25] Fukuyama, H. and Lee, P. A., Phys. Rev. B 17 (1978) 476.

LEE, P. A. and RICE, T. M., Phys. Rev. B 19 (1979) 3970. 